\title{
A Conjecture of Paul Erdös Concerning Gaussian Primes
}

\author{
By J. H. Jordan and J. R. Rabung
}

\begin{abstract}
Paul Erdös has conjectured that you can stroll from the origin of the complex plane to infinity using the Gaussian primes as stepping stones and be required only to take steps of finite length. This paper establishes that steps of length 4 would be required to make the journey.
\end{abstract}

It is well known that if $P_{n}$ is the $n$th prime then the values $\left\{P_{n}-P_{n-1}\right\}$ are without bound. That is, lim sup $\left\{P_{n}-P_{n-1}\right\}=\infty$. Put in every day language this says that there exists arbitrarily large intervals which have no primes. This in some sense says that if you used the primes as stepping stones and attempted to walk from 2 out the real line you would from time to time be called on to take longer and longer steps.

Recently Basil Gordon communicated to the authors a conjecture of Paul Erdös concerning the two-dimensional generalization of this problem. The conjecture is that you can use the Gaussian primes as stepping stones, start in the vicinity of the origin and stroll out to infinity never being required to take a step of length more than $m$. Put more precisely: (Erdös' conjecture) there is an $M$ and a sequence of Gaussian primes $\left\{\gamma_{j}\right\}_{1}^{\infty}$, such that $\left|\gamma_{1}\right|<M,\left|\gamma_{j}-\gamma_{j-1}\right|<M$ and $\lim \left|\gamma_{j}\right|=\infty$.

Due to the symmetry of the Gaussian primes with respect to $x$-axis, $y$-axis, $x=y$ and $x=-y$, the Erdös conjecture can be restricted to $0 \leqq \arg \left(\gamma_{j}\right) \leqq \pi / 4$ with no strengthening of the problem.

Since all $\gamma_{j}$ except $1+i$ are odd it follows $\left|\gamma_{j}-\gamma_{j-1}\right| \geqq \sqrt{ } 2$.

We wish to report that if the conjecture is true then $M \geqq 4$.

To illustrate our procedure we begin by establishing that $M \geqq 2$. To show this we list a trail of composites that divide the points around the origin from the rest of the plane. If $\alpha$ is a prime outside this region and $\beta$ is a prime inside, then $|\alpha-\beta| \geqq 2$.

The vertices of the trail are $\{12,12+5 i, 9+5 i, 9+9 i\}$. There is one place that the trail passes through a "strait" of length 2 .

We next show a trail illustrating $M \geqq 2 \sqrt{ } 2$.

The vertices of the trail are $\{42,42+3 i, 43+3 i, 43+7 i, 42+7 i, 42+12 i$, $40+12 i, 40+16 i, 43+16 i, 43+18 i, 38+18 i, 38+22 i, 37+22 i, 37+27 i$, $38+27 i, 38+38 i\}$. There are two "straits" of width $2 \sqrt{ } 2$ on this trail at $43+18 i$ and $38+33 i$.

Our next stage is to show that $M \geqq \sqrt{ } 10$. The vertices of the trail that shows this are $\{68,68+3 i, 67+3 i, 67+17 i, 69+17 i, 69+23 i, 77+23 i, 77+29 i$, $82+29 i, 82+34 i, 83+34 i, 83+40 i, 85+40 i, 85+41 i, 85+42 i, 84+42 i$, $84+57 i, 86+57 i, 86+59 i, 77+59 i, 77+71 i, 76+71 i, 76+76 i$.

There are 17 "straits" of width $\sqrt{ } 10$ on this trail.

Finally the trail illustrating $M \geqq 4$.

Received June 4, 1969, revised July 28, 1969.

AMS Subject Classifications. Primary 1066, 1063.

Key Words and Phrases. Gaussian integers, Gaussian primes, distribution of Gaussian primes. 
We list the vertices of the trail.

$\begin{array}{lllll}868+0 i & 984+169 i & 956+336 i & 900+469 i & 904+670 i \\ 868+26 i & 984+180 i & 956+342 i & 900+484 i & 904+683 i \\ 863+26 i & 986+180 i & 950+342 i & 905+484 i & 911+683 i \\ 863+42 i & 986+190 i & 950+345 i & 905+494 i & 911+698 i \\ 861+42 i & 978+190 i & 938+345 i & 906+494 i & 891+698 i \\ 861+47 i & 978+202 i & 938+341 i & 906+508 i & 891+692 i \\ 864+47 i & 979+202 i & 927+341 i & 905+508 i & 870+692 i \\ 864+49 i & 979+214 i & 927+342 i & 905+516 i & 870+685 i \\ 859+49 i & 977+214 i & 924+342 i & 918+516 i & 861+685 i \\ 859+70 i & 977+216 i & 924+349 i & 918+515 i & 861+680 i \\ 864+70 i & 978+216 i & 918+349 i & 920+515 i & 853+680 i \\ 864+75 i & 978+224 i & 918+345 i & 920+533 i & 853+703 i \\ 882+75 i & 972+224 i & 902+345 i & 917+533 i & 857+703 i \\ 882+87 i & 972+243 i & 902+354 i & 917+545 i & 857+714 i \\ 891+87 i & 968+243 i & 892+354 i & 920+545 i & 855+714 i \\ 891+103 i & 968+252 i & 892+372 i & 920+558 i & 855+723 i \\ 897+103 i & 945+252 i & 893+372 i & 909+558 i & 863+723 i \\ 897+109 i & 945+261 i & 893+381 i & 909+568 i & 863+732 i \\ 903+109 i & 952+261 i & 892+381 i & 923+568 i & 853+732 i \\ 903+112 i & 952+266 i & 892+387 i & 923+590 i & 853+737 i \\ 906+112 i & 959+266 i & 898+387 i & 907+590 i & 847+737 i \\ 906+126 i & 959+270 i & 898+416 i & 907+595 i & 847+740 i \\ 949+126 i & 974+270 i & 892+416 i & 909+595 i & 834+740 i \\ 949+127 i & 974+283 i & 892+425 i & 909+602 i & 834+748 i \\ 953+127 i & 977+283 i & 887+425 i & 900+602 i & 819+748 i \\ 953+124 i & 977+289 i & 887+424 i & 900+615 i & 819+765 i \\ 967+124 i & 976+289 i & 883+424 i & 898+615 i & 814+765 i \\ 967+131 i & 976+299 i & 883+429 i & 898+623 i & 814+783 i \\ 972+131 i & 978+299 i & 877+429 i & 902+623 i & 803+783 i \\ 972+137 i & 978+304 i & 877+444 i & 902+642 i & 803+801 i \\ 982+137 i & 977+304 i & 882+444 i & 896+642 i & 801+801 i \\ 982+162 i & 977+316 i & 882+458 i & 896+660 i & \\ 978+162 i & 968+316 i & 897+458 i & 894+660 i & \\ 978+169 i & 968+336 i & 897+469 i & 894+670 i & \\ +52 i & & & \end{array}$

There are 43 "straits" of width 4 on this trail. Some of these straits can be avoided by zig-zagging the path.

A sequence of Gaussian primes starting with $2+i$ and terminating with 985 $+182 i$ exists with the absolute value of the difference of consecutive elements $\leqq \sqrt{ } 10$. This indicates that any other trail which illustrates $M \geqq 4$ would have to encircle this sequence. Notice our trail goes through point $986+182 i$. We were forced to use more than 100 times as many data to establish $M=4$ as we needed to establish $M=\sqrt{ } 10$. This leads us to believe the conjecture is true.

The next problem to be attacked would be to try to show $M \geqq \sqrt{ } 18$ and then $M \geqq 2 \sqrt{ } 5$ and $M \geqq \sqrt{ } 26$. 
We utilized the IBM 360 to generate and plot Gaussian primes and then manually examined the output to determine the trail.

It may be possible for the computer to locate these trails but we were unable to establish an effective program that would operate within budgetary limits.

We wish to express our appreciation to the Pennsylvania State University for use of their computing facilities.

Washington State University

Pullman, Washington 99163

U. S. Naval Research Laboratory

Washington, D. C. 20390 